Open Access

\title{
Predictors and patterns of recurrence after curative liver resection in intrahepatic cholangiocarcinoma, for application of postoperative radiotherapy: a retrospective study
}

Eunmi Gil ${ }^{1}$, Jae-Won Joh ${ }^{2^{*}+}$, Hee Chul Park ${ }^{3^{*+}}$, Jeong $\| Y_{u^{3}}$, Sang Hoon Jung ${ }^{3}$ and Jong Man $\mathrm{Kim}^{2}$

\begin{abstract}
Background: The majority of patients with intrahepatic cholangiocarcinoma (IHCC) who undergo complete tumor resection subsequently develop tumor recurrence. The objectives of this study were to determine the risk factors for IHCC recurrence after curative (RO) liver resection and to identify the feasibility about postoperative adjuvant radiation therapy (RT).

Methods: We retrospectively reviewed patients who underwent liver resection for IHCC between April 1995 and December 2012 at Samsung Medical Center. Cox regression analysis was performed to determine risk factors of recurrence. Patients with a recurrence in remnant liver within $2 \mathrm{~cm}$ from the resection margin, with or without locoregional lymph node (LN) metastases, were considered as potential RT candidates. Center-of-mass (COM) distances between the recurrent cancers and the cut surface were measured with MATLAB.

Results: We included 153 out of 198 patients who underwent partial liver resection for IHCC. About two thirds ( $n=93$, $60.8 \%$ ) of patients developed recurrent disease. The median recurrence-free survival (RFS) was 14 months (range, 0-204). Tumor size $\geq 4.0 \mathrm{~cm}$, LN metastasis and multiple tumors were significant predictors of IHCC recurrence on multivariate analysis. Tumor size $\geq 5.0 \mathrm{~cm}$ was the only factor associated with recurrence beyond the RT field in patients with recurrence. Among 93 patients with recurrence, 16 (17.2\%) patients were recurred in the RT field.

Conclusion: After curative resection in IHCC, more than $60 \%$ of patients recurred, and among recurred patients, $17.2 \%$ were recurred within the RT field. Consequently, for control of locoregional recurrence, adjuvant RT could be carefully considered in patients with recurrence factors. Especially, patients with a tumor size larger than $5 \mathrm{~cm}$ should be judiciously selected for adjuvant RT.
\end{abstract}

Keywords: Intrahepatic cholangiocarcinoma, Recurrence, Hepatectomy, Adjuvant radiotherapy

\section{Background}

Cholangiocarcinoma (CC) is the second most common primary hepatic malignancy, after hepatocellular carcinoma, and is divided into three general categories based on the origin of disease, intrahepatic, hilar, or distal CC

\footnotetext{
* Correspondence: jwjoh@skku.edu; hee.ro.park@samsung.com

${ }^{\dagger}$ Equal contributors

${ }^{2}$ Department of Surgery, Samsung Medical Center, Sungkyunkwan University School of Medicine, 50 Irwon-dong, Gangnam-gu, Seoul 135-170, South Korea

${ }^{3}$ Department of Radiation Oncology, Samsung Medical Center, Sungkyunkwan University School of Medicine, 50 Irwon-dong, Gangnam-gu, Seoul 135-170, South Korea

Full list of author information is available at the end of the article
}

$[1,2]$. Although hilar $\mathrm{CC}$ remains the most common type, the incidence of intrahepatic cholangiocarcinoma (IHCC) is rising [3-5], and IHCC currently accounts for $20 \%$ of all CC [5].

IHCC has a dismal prognosis with limited treatment options and a very high rate of recurrent or metastatic disease $[1,2]$. Indeed, the overall mortality rate in IHCC approaches its incidence [6]. Currently, surgical resection offers the only chance for cure. However, as the disease lacks symptoms until late in its course, the majority of IHCC patients have unresectable tumors at diagnosis, and less than $50 \%$ of patients with IHCC are surgical 
candidates [2, 7-11]. Even after complete resection, recurrence rates approach $52 \%$, with 5 -year postresection survival rates ranging from 8 to $44 \%$, indicating that resection alone is not sufficient for most patients $[1,6-9,12,13]$.

Repeated surgery is limited by patients' comorbidities or by poor functional hepatic reserve. In patients with recurrence, palliative treatments such as radiation and systemic chemotherapy are the only options. However, current studies testing the role of adjuvant chemotherapy in IHCC are limited [14-16]. While adjuvant radiation therapy may improve survival in patients with microscopically positive margins, this remains controversial [17-21] and no guidelines for adjuvant therapy for IHCC exist [22]. The benefit of adjuvant RT in resected IHCC will undoubtedly depend on appropriate patient selection. The purpose of this study is to identify a patient subset at high risk for locoregional tumor recurrence after curative resection and to identify the feasibility about postoperative adjuvant radiation therapy.

\section{Methods}

\section{Patient selection and data collection}

We retrospectively reviewed patients 18 years of age or older with IHCC who underwent liver resection at Samsung Medical Center between April 1995 and December 2012. We excluded patients with a history of other malignancies, and those who had neoadjuvant therapy for IHCC, M1 disease, and R2 or R1 resection. Postoperatively, patients were followed with serial computed tomography $(\mathrm{CT})$ scans and serial serum tumor marker (CA 19-9 and CEA) levels.

Pathology reports were reviewed for important prognostic factors including tumor size, subtype, number and histology, margin status, lymph node (LN) involvement, and the presence of lymphovascular invasion (LVI) and perineural invasion (PNI), which are known important factors for tumor recurrence and patient survival [23-25]. Satellite nodules were considered as multiple tumors. Patients who did not undergo LN dissection were regarded as node-negative, as this is consistent with clinical practice patterns. The first postoperative follow-up evaluation was usually 1 month after the operation, and subsequent visits were performed according to the surgeon's discretion. Overall survival was ascertained through the clinical follow-up documented in each patient's medical record.

\section{Recurrence classification}

The regions of the recurrent cancers and the cut surface were delineated on the follow-up CT or magnetic resonance (MR) using Eclipse ver. 10.0 (Varian, Palo
Alto, CA), and the structure files in digital imaging and communications in medicine (DICOM), including the regions, were exported to an in-house program in MATLAB (Mathworks, Natick, NA). The closest, farthest, and center-of-mass (COM) distances between the recurrent cancers and the cut surface were measured with the program. Curability was determined according to the R-classification of the International Union Against Cancer as R0, no residual tumor; R1, presence of microscopic disease despite removal of all identifiable tumor; and R2, tumor left macroscopically in situ.

Regional LNs of IHCC included the hilar LNs. And according to LN drainage, tumors in the right liver (segments 5-8) included pancreaticoduodenal LNs, and tumors in the left liver (segments 2-4) included gastrohepatic LNs. Diseases that spread to the celiac, periaortic, or caval LNs were usually considered distant metastases (M1 node), but clinical radiation therapy (RT) fields generally encompassed periaortic or caval LNs around celiac LNs.

Therefore, local recurrence in the present study was defined as any recurrence within the potential planning target volume (PTV) in RT fields. We defined the potential clinical target volume (CTV) in the present study as remnant liver within $1 \mathrm{~cm}$ from resection margin, perihilar, periduodenal, peripancreatic, celiac, caval, and periaortic (from $1 \mathrm{~cm}$ above of celiac axis to left renal artery bifurcation) LN area. PTV was decided with an additional $1 \mathrm{~cm}$ margin for setup and respiration uncertainties. We assumed radiation delivered as a 3-D conformal technique, and the remnant liver, kidneys, stomach, duodenum, other bowel, and spinal cord were considered as organs at risk. Hypothetical CTV, RT fields were displayed in Fig. 1. And, the authors hypothesized patients who recurred in this area which is remnant liver within $2 \mathrm{~cm}$ of the COM with or without locoregional LN metastasis as potential RT candidates.

\section{Statistical analysis}

Summary statistics are reported as total and percentage for categorical variables and as median values and range for continuous variables. Survival and recurrence rates were calculated by the Kaplan-Meier method. Cox regression analysis was performed to determine recurrence factors. If data for a variable were missing in $>10 \%$ of cases, the variable was not used in the analysis. Factors significant at a level of $p<0.1$ were included in the multivariate analysis. A receiver operating characteristics (ROC) curve was used to identify optimal cutoff points for each marker. All statistical analyses were carried out using IBM SPSS 


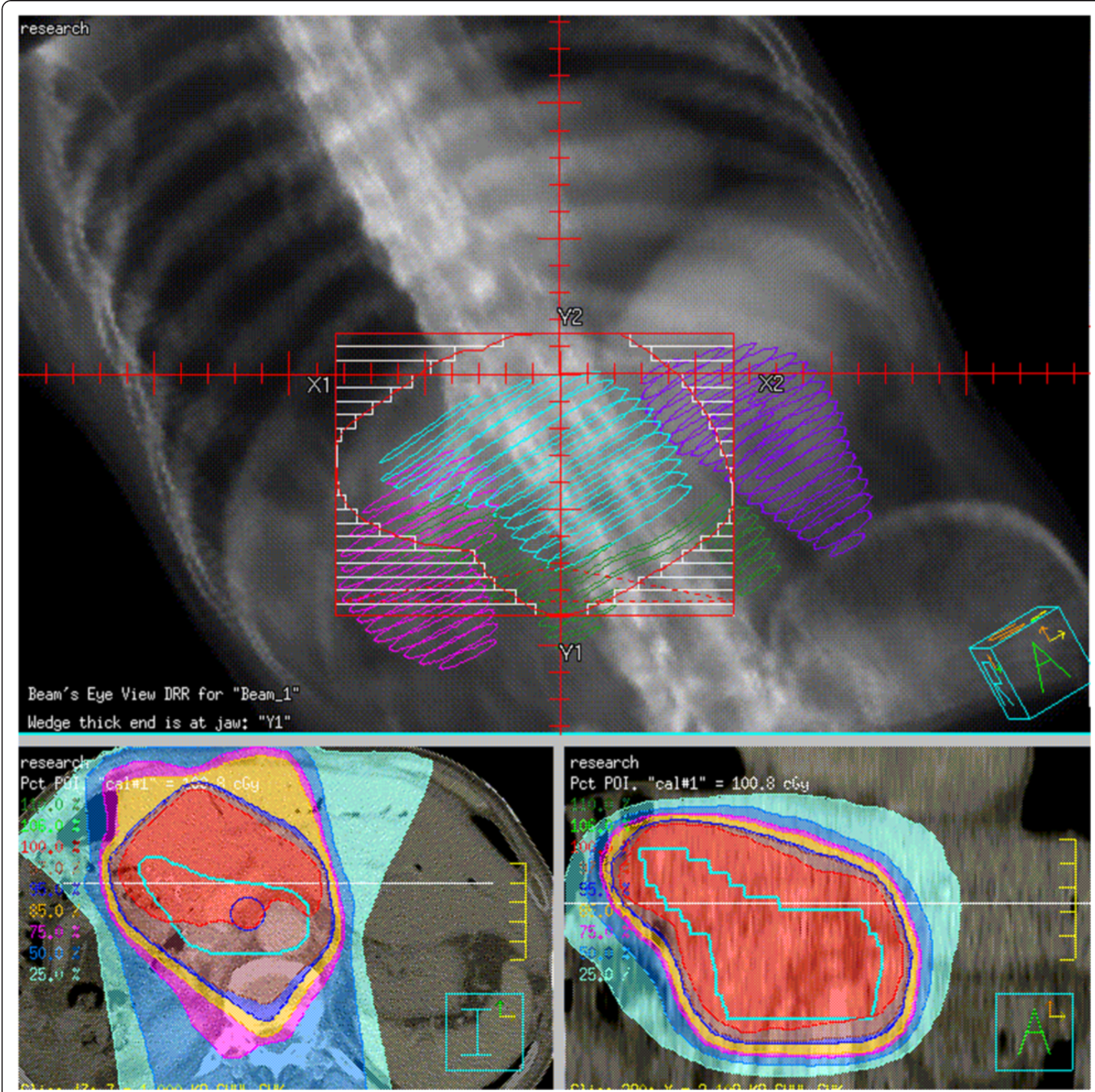

Fig. 1 Hypothetical adjuvant radiotherapy fields in intrahepatic cholangiocarcinoma. Potential clinical target volume (CTV) is defined as remnant liver within $1 \mathrm{~cm}$ from resection margin, perihilar, periduodenal, peripancreatic, celiac, caval, and periaortic (from $1 \mathrm{~cm}$ above of celiac axis to left renal artery bifurcation) lymph node area

statistics version 22. A $p$ value of less than 0.05 was considered to be statistically significant.

\section{Results}

Patients and tumor characteristics

A total of 198 patients underwent partial liver resection for IHCC between April 1995 and December 2012. We excluded 19 patients who had another previous malignancy, four patients who had preoperative transarterial chemoembolization (TACE), three patients who had neoadjuvant chemotherapy before hepatectomy, a patient with bone metastasis who underwent palliative hepatectomy, two patients with R2 disease, and 12 patients with R1 disease. Four patients were lost to followup (Fig. 2).

The final study population $(n=153)$ had resected IHCC only, with a median age of 59 years (range, 3780 years); 96 patients $(62.7 \%)$ were male. Operative and pathologic variables are summarized in Table 1 . The majority $(90.1 \%)$ of patients required a hemihepatectomy, 


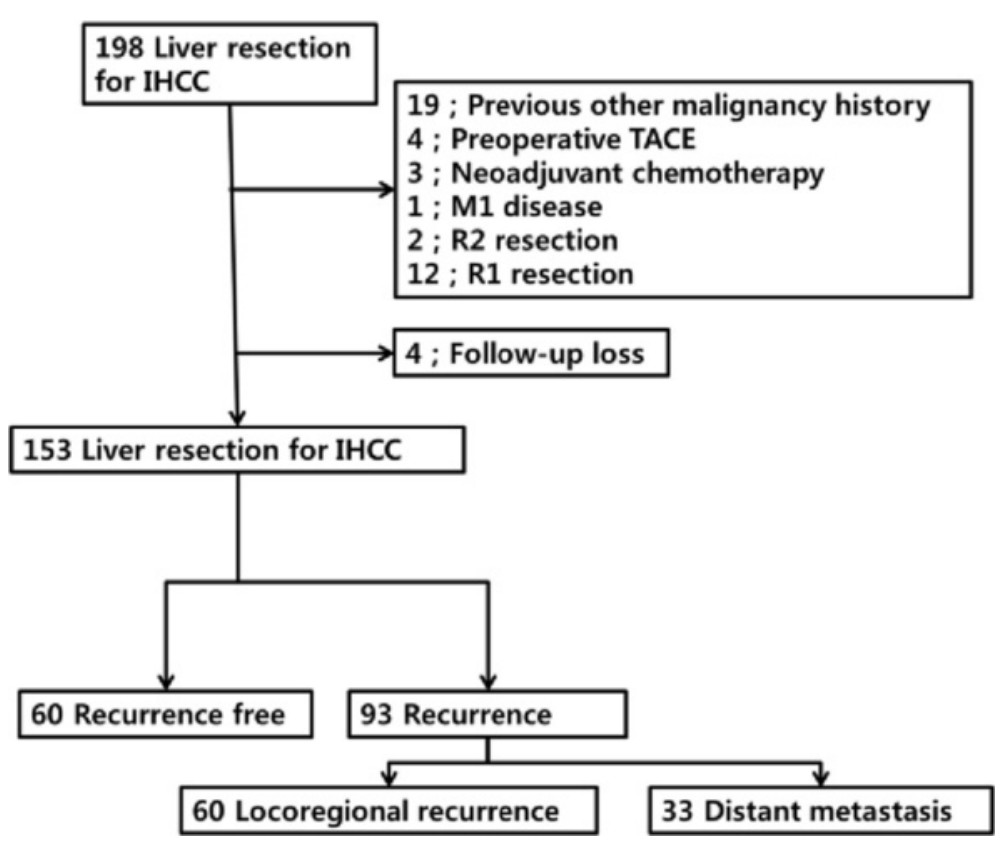

Fig. 2 Patient selection and recurrence after liver resection for intrahepatic cholangiocarcinoma

extended resection, or multiple segmental resection. Eighty-one patients (52.9\%) underwent lymphadenectomy. The median tumor size of the dominant lesion was $4.5 \mathrm{~cm}$ (range, $0.5-14.0 \mathrm{~cm}$ ). LN metastasis occurred in 38 patients $(24.8 \%)$, and 37 patients (24.2\%) had multiple confirmed tumors in the final pathologic report. One hundred seventeen patients $(76.5 \%)$ had the mass-forming subtype of IHCC, while 36 patients (23.5\%) had the periductal infiltrating type or combined both types. LVI was found in $35.9 \%$ of patients, and perineural invasion (PNI) was found in $23.5 \%$. Two patients had adjuvant chemoradiation therapy after curative resection.

\section{Recurrence and survival}

After a median follow-up of 21 months (range, 1204 months), recurrence had developed in 93 patients (60.8\%) (Fig. 2). Among 93 patients with recurrence, 60 patients $(64.5 \%)$ had recurrences in the remnant liver and/or locoregional LNs. Thirty-three patients (35.5\%) were diagnosed with distant metastasis. The most common distant metastasis site was the lung. Eleven patients recurred in the liver, locoregional LN, and distant metastasis sites. Figure 3 shows recurrence patterns of IHCC. The median recurrence-free survival (RFS) was 14 months (range, 0-204), and the median overall survival (OS) was 35 months (range, 1-204). Tumor size was an important factor for recurrence based on univariate Cox regression analysis $(p<0.001)$. LN metastasis, tumor subtype, multiple tumors, LVI, PNI, and prothrombin timeinternational normalized ratio (PT-INR) were recurrence factors based on univariate analysis. The optimal cutoff for tumor size as a recurrence risk factor was $\geq 4.0 \mathrm{~cm}(p<$ 0.001 AUC 0.740, CI 0.661-0.819). Tumor size $\geq 4.0 \mathrm{~cm}$ $(p<0.001), \mathrm{LN}$ metastasis $(p=0.006)$, and multiple tumors $(p=0.015)$ were significant predictors of IHCC recurrence on multivariate analysis (Table 2). Predictors of survival were similar to those for recurrence. On univariate analysis, INR, tumor subtype, tumor size, multiple tumors, LVI, PNI, and LN metastasis were risk factors. Tumor size $\geq 4.0 \mathrm{~cm}(p<0.001)$, LN metastasis $(p<0.01)$, multiple tumors $(p=0.014)$, and LVI $(p=$ $0.015)$ were risk factors on multivariate analysis (Table 3).

\section{Potential adjuvant RT candidates}

Figure 3 shows recurrence in remnant liver and/or locoregional LNs of IHCC. Among 93 patients with recurrence, 60 (64.5\%) recurred in remnant liver and/or locoregional LNs and only 16 patients (17.2\%; gray area in Fig. 4) were included in the hypothetical RT field. Forty-four patients recurred in remnant liver more than $2 \mathrm{~cm}$ far from the cut surface with or without locoregional LN metastasis, in other words, recurred beyond the RT field (white area in Fig. 4). Among the 16 patients, two patients recurred in the liver, 12 recurred in locoregional LNs, and two patients recurred in both the liver and locoregional LNs. Among patient recurred, tumor size $(p<0.001)$ was the only predictive factor of recurrence beyond the hypothetical RT field. The optimal cutoff point for tumor size as a predictor of recurrence beyond the hypothetical RT field was $\geq 5.0 \mathrm{~cm}$ ( $p=0.045$ 
Table 1 Clinicopathologic characteristics of patients with intrahepatic cholangiocarcinoma $(n=153)$

\begin{tabular}{|c|c|c|}
\hline Variable & Patients, $n(\%)$ & Median (range) \\
\hline Age & & $59(37-80)$ \\
\hline Male & $96(62.7)$ & \\
\hline \multicolumn{3}{|l|}{ Operative characteristics } \\
\hline \multicolumn{3}{|l|}{ Type of resection } \\
\hline Wedge resection & $3(2.0)$ & \\
\hline Segmentectomy & $11(7.2)$ & \\
\hline Multiple segmentectomy & $25(16.3)$ & \\
\hline Hemihepatectomy & $92(60.1)$ & \\
\hline Extended hemihepatectomy & $22(14.4)$ & \\
\hline \multicolumn{3}{|l|}{ Pathologic characteristics } \\
\hline Tumor size, cm & & $4.5(0.5-14.0)$ \\
\hline Tumor size $\geq 4 \mathrm{~cm}$ & $97(63.4)$ & \\
\hline Multiple tumors & $37(24.2)$ & \\
\hline Lymphadenectomy performed & $81(52.9)$ & \\
\hline LN positive disease & $38(24.8)$ & \\
\hline Number of LN retrieved & & $7(1-44)$ \\
\hline Number of positive nodes & & $0(0-22)$ \\
\hline \multicolumn{3}{|l|}{ Subtype } \\
\hline Mass forming & $117(76.5)$ & \\
\hline Periductal infiltrative & $36(23.5)$ & \\
\hline \multicolumn{3}{|l|}{ Histology } \\
\hline Adenocarcinoma & $140(91.5 \%)$ & \\
\hline Non-adenocarcinoma & $13(8.5 \%)$ & \\
\hline Lymphovascular invasion & $55(35.9)$ & \\
\hline Perineural invasion & $36(23.5)$ & \\
\hline
\end{tabular}

LN lymph node
AUC 0.628, CI 0.504-0.751) $(n=93)$ (Table 4). There were no statistically significant factors between four patients recurred in remnant liver within $2 \mathrm{~cm}$ of $\mathrm{COM}$ and other recurred patients.

\section{Discussion}

Surgical resection is the only potential cure for IHCC. Although resectability of IHCC has improved recently [10], the success rate remains poor [10,11]. Even in patients who are able to undergo surgical resection, positive margins are common $[10,11]$. The use of $\mathrm{R} 0$ resection provides the best chance for cure but is only achievable in about $30 \%$ of patients at presentation [11]. Studies have demonstrated that patients who had an R1 resection had a survival similar to those who were treated palliatively (5 vs. 7 months) and that local recurrence is a major problem in patients with $\mathrm{R} 1 \mathrm{re}$ sections $[11,26]$.

Even after R0 resection, many studies reported recurrence rates more than $50 \%$, representing that resection alone is not sufficient for most patients $[1,6-9,12,13]$. Like other studies, our data shows $60.8 \%$ recurrence rate in IHCC after R0 resection. In patients with recurrence, palliative treatments such as radiation and systemic chemotherapy could be considered. Earlier reports of postoperative adjuvant therapy for the treatment of biliary tract carcinoma have indicated the potential usefulness of radiotherapy and chemotherapy [27-30]. However, current studies testing the role of adjuvant chemotherapy in IHCC are limited [14-16]. While adjuvant radiation therapy may improve survival in patients with microscopically positive margins, this remains controversial [17-21]. Moreover, nearly all these studies were based on cases of noncurative resection and were experimental reports rather than clinical trial reports. In addition, some hilar $\mathrm{CC}$ have been misclassified as

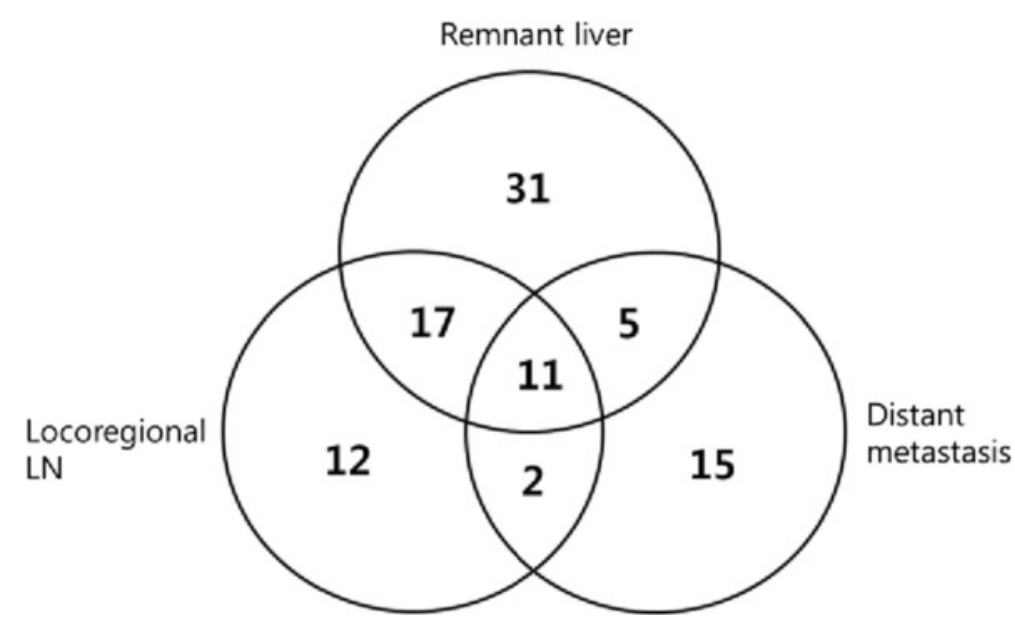

Fig. 3 Recurrence pattern of intrahepatic cholangiocarcinoma $(n=93)$ 
Table 2 Predictors of recurrence after resection of intrahepatic cholangiocarcinoma $(n=153)$

\begin{tabular}{|c|c|c|c|c|}
\hline \multirow[t]{2}{*}{ Characteristic } & \multicolumn{2}{|l|}{$\underline{\text { Univariate }}$} & \multicolumn{2}{|l|}{ Multivariate } \\
\hline & $\mathrm{HR}(95 \% \mathrm{Cl})$ & $p$ value & $\mathrm{HR}(95 \% \mathrm{Cl})$ & $p$ value \\
\hline Sex & $1.046(0.688-1.589)$ & 0.835 & & \\
\hline Age & $1.011(0.988-1.034)$ & 0.360 & & \\
\hline PT-INR & $7.894(1.041-59.841)$ & 0.046 & & \\
\hline Type of resection & & 0.483 & & \\
\hline Wedge resection & $0.917(0.223-9.767)$ & 0.904 & & \\
\hline Segmentectomy & $1.264(0.601-2.661)$ & 0.537 & & \\
\hline Multiple segmentectomy & $0.729(0.399-1.335)$ & 0.306 & & \\
\hline Hemihepatectomy & 1 & & & \\
\hline Extended hemihepatectomy & 1.419 (0.799-2.518) & 0.232 & & \\
\hline Tumor subtype & $0.386(0.221-0.676)$ & 0.001 & & \\
\hline Histology & $0.394(0.144-1.075)$ & 0.069 & & \\
\hline Tumor size $\geq 4.0 \mathrm{~cm}$ & $2.932(1.830-4.696)$ & $<0.001$ & $2.522(1.556-4.088)$ & $<0.001$ \\
\hline Multiple tumors & $2.020(1.279-3.191)$ & 0.003 & $1.815(1.125-2.927)$ & 0.015 \\
\hline LVI & $1.866(1.232-2.826)$ & 0.003 & & \\
\hline $\mathrm{PNI}$ & $1.825(1.156-2.880)$ & 0.010 & & \\
\hline LN metastasis & $2.277(1.424-3.642)$ & 0.001 & $2.004(1.221-3.289)$ & 0.006 \\
\hline Resection margin & $0.988(0.972-1.044)$ & 0.144 & & \\
\hline HBV & $1.135(0.685-1.881)$ & 0.623 & & \\
\hline $\mathrm{HCV}$ & $1.550(0.624-3.849)$ & 0.345 & & \\
\hline
\end{tabular}

LVI lymphovascular invasion, $P N I$ perineural invasion, $H B V$ hepatitis $B$ virus, $H C V$ hepatitis $C$ virus, $P T-I N R$ prothrombin time-international normalized ratio

Table 3 Predictors of survival after resection of intrahepatic cholangiocarcinoma $(n=153)$

\begin{tabular}{|c|c|c|c|c|}
\hline \multirow[t]{2}{*}{ Characteristic } & \multicolumn{2}{|l|}{ Univariate } & \multicolumn{2}{|l|}{ Multivariate } \\
\hline & $\mathrm{HR}(95 \% \mathrm{Cl})$ & $p$ value & $\mathrm{HR}(95 \% \mathrm{Cl})$ & $p$ value \\
\hline Sex & $0.873(0.568-1.344)$ & 0.538 & & \\
\hline Age & $1.011(0.988-1.036)$ & 0.347 & & \\
\hline PT-INR & 24.618 (3.449-161.735) & 0.001 & & \\
\hline Type of resection & & 0.137 & & \\
\hline Wedge resection & $0.450(0.062-3.258)$ & 0.429 & & \\
\hline Segmentectomy & $0.949(0.432-2.087)$ & 0.897 & & \\
\hline Multiple segmentectomy & $0.655(0.343-1.252)$ & 0.201 & & \\
\hline Hemihepatectomy & 1 & & & \\
\hline Extended hemihepatectomy & 1.677 (0.969-2.903) & 0.065 & & \\
\hline Tumor subtype & $0.367(0.207-0.654)$ & 0.001 & $0.536(0.297-0.968)$ & 0.039 \\
\hline Histology & $0.680(0.314-1.475)$ & 0.329 & & \\
\hline Tumor size $\geq 4.0 \mathrm{~cm}$ & $3.162(1.928-5.184)$ & $<0.001$ & $2.539(1.512-4.264)$ & $<0.001$ \\
\hline Multiple tumor & $2.475(1.585-3.864)$ & $<0.001$ & $1.782(1.122-2.828)$ & 0.014 \\
\hline LVI & $2.299(1.520-3.475)$ & $<0.001$ & $1.684(1.107-2.564)$ & 0.015 \\
\hline $\mathrm{PNI}$ & $1.869(1.181-2.957)$ & 0.008 & & \\
\hline LN metastasis & $3.902(2.499-6.091)$ & $<0.001$ & $3.567(2.240-5.680)$ & $<0.001$ \\
\hline Resection margin & $0.991(0.974-1.008)$ & 0.294 & & \\
\hline HBV & 1.165 (0.695-1.955) & 0.562 & & \\
\hline $\mathrm{HCV}$ & $1.226(0.496-3.026)$ & 0.659 & & \\
\hline
\end{tabular}

LVI lymphovascular invasion, $P N I$ perineural invasion, $H B V$ hepatitis B virus, $H C V$ hepatitis $\mathrm{C}$ virus, $P T$-INR prothrombin time-international normalized ratio 


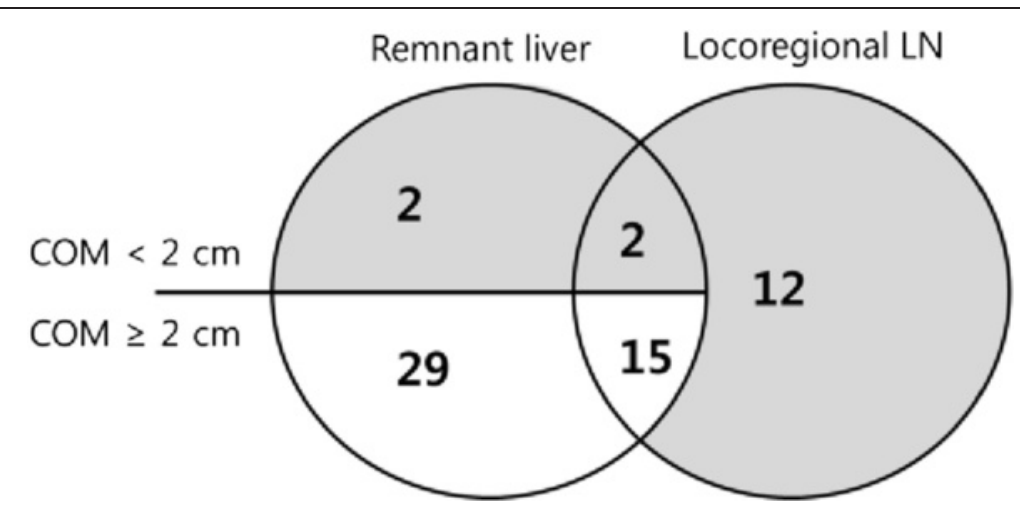

Fig. 4 Recurrence in remnant liver and/or locoregional LNs of intrahepatic cholangiocarcinoma $(n=60)$. Patients in gray area $(n=16)$ included hypothetical radiation therapy field

IHCC, thereby affecting analysis [21]. Thus, the benefit of adjuvant therapy for carcinoma of the biliary tract has not yet been clarified, and no guidelines for adjuvant therapy for IHCC exist [22]. The benefit of postoperative adjuvant RT will depend on appropriate patient selection. So, authors determined to identify a patient subset at high risk for locoregional tumor recurrence after R0 resection and a subgroup among these patients who could be covered within the RT field.

As mentioned above, some hilar $\mathrm{CC}$ have been misclassified as IHCC and many studies included R1 resection also. However, this report included only patients with pathologically confirmed IHCC with R0 resection. Patients with perihilar or distal CC, history of other malignancy, history of neoadjuvant therapy (TACE and chemotherapy), M1 disease, and R1 and R2 diseases were excluded from this analysis. After curative

Table 4 Predictors of recurrence beyond the radiation therapy field in recurrent patients $(n=93)$

\begin{tabular}{lll}
\hline Characteristic & HR $(95 \% \mathrm{Cl})$ & $p$ value \\
\hline Sex & $1.194(0.745-1.914)$ & 0.462 \\
Age & $0.976(0.948-1.004)$ & 0.095 \\
PT-INR & $7.155(0.498-102.760)$ & 0.148 \\
Tumor subtype & $0.566(0.294-1.092)$ & 0.090 \\
Histology & $0.753(0.234-2.426)$ & 0.635 \\
Tumor size & $1.204(1.101-1.317)$ & $<0.001$ \\
Tumor size $\geq 5.0 \mathrm{~cm}$ & $3.140(1.890-5.217)$ & $<0.001$ \\
LVI & $1.288(0.810-2.049)$ & 0.284 \\
PNI & $1.239(0.745-2.060)$ & 0.409 \\
LN metastasis & $1.567(0.924-2.655)$ & 0.095 \\
Resection margin & $0.985(0.968-1.004)$ & 0.113 \\
HBV & $0.806(0.448-1.450)$ & 0.471 \\
HCV & $1.236(0.496-3.085)$ & 0.649 \\
\hline
\end{tabular}

LVI lymphovascular invasion, $P N I$ perineural invasion, $H B V$ hepatitis B virus, $H C V$ hepatitis $C$ virus, $P T-I N R$ prothrombin time-international normalized ratio resection, two patients had adjuvant chemoradiation therapy. A patient was 53 years old, female, and with multiple IHCC. The maximum tumor size was $5.5 \mathrm{~cm}$. IHCC recurred after 15 months of surgery. Another patient was 48 years old, female, and with multiple IHCC. The maximum tumor size was $7.8 \mathrm{~cm}$, and LN metastasis exists during surgery. IHCC recurred after a month, and she died after 4 months of surgery. Because only two patients had adjuvant treatment, it was impossible to analyze statistical significance. And after IHCC recurrence, 12 patients had palliative chemoradiation therapy and a patient had palliative RT. There was no significant difference about survival between two groups that had palliative treatment or not (data not shown).

Jarnagin et al. [31] reported about adjuvant treatment after surgery of extrahepatic cholangiocarcinoma. After surgery, locoregional recurrence is a predominant pattern of failure even with R0 resection, and as local failure can be associated with liver failure and death, this argues for adjuvant radiation and chemoradiation therapy to prevent local recurrence. Shinohara et al. [21] reported radiation used adjuvantly with surgery was associated with an improved median survival compared with radiation alone and was associated with a $9.3 \%$ reduction in the risk of death. However, although radiation did prolong survival in these patients, it did not appear to cure their disease. And these studies did not specify the criteria about adjuvant treatment candidates after curative resection. The benefit of adjuvant RT in resected IHCC will depend on appropriate patient selection.

Pathologic factors that predict increased rates of recurrence and worse outcomes include multiple hepatic tumors, nodal involvement, and vascular invasion, which was reflected in the current American Joint Committee on Cancer (AJCC) 7th edition staging system [8, 32]. Although LN stats have been strongly associated with prognosis in almost all studies [33-35], data on tumor size have been more disparate, with some studies finding 
no effect of tumor size [36-38] and other larger studies showing that size affected survival [33-35]. Previously, the failure to identify an effect of tumor size on OS may be explained by the limited number of patients with small (e.g., $<5 \mathrm{~cm}$ ) tumors included in many prior studies. Our study found a tumor size larger than $4 \mathrm{~cm}$ is an important risk factor of recurrence and survival. Moreover, tumor size larger than $5 \mathrm{~cm}$ is the only risk factor of recurrence beyond the hypothetical RT field. Previous studies did not consider about tumor size as an indication of adjuvant treatment. Future studies about adjuvant RT and the AJCC staging system may need to reconsider the impact of tumor size on prognosis and should include tumor size as selection criteria of adjuvant treatment.

Our findings should be interpreted in view of certain limitations. First, retrospective study and the sample size were small. Moreover, this study was accrued over a long period (1995-2012), while both diagnostic and therapeutic procedures for IHCC have changed notably over these years. There may be a selection bias in how patients were chosen for surgical therapy. Therefore, further multicentric prospective and randomized clinical trials are needed to determine the usefulness of adjuvant $\mathrm{RT}$ to control locoregional recurrence after R0 resection.

With IHCC, NCCN recommendations include observation for R0 resection or some type of adjuvant therapy for R1 or R2 resections. However, this study demonstrates that tumor recurrence is common (60.8\%) after complete resection (R0) of IHCC. In this light, after R0 curative liver resection, observation is not appropriate. For control of recurrence, aggressive adjuvant treatment should be considered even if curative resection is achieved. In our study, tumor size $\geq 4.0 \mathrm{~cm}$, LN metastasis, and multiple tumors predicted recurrence after curative resection. Among recurred patients, locoregional recurrence covered in the hypothetical RT field occurred less than $20 \%$. Even though a small portion of patients recurred in hypothetical RT field, authors consider postoperative RT might be a useful adjuvant modality because RT could sterilize the field; as a result, it could lead to lower the recurrence rate by lowering in and out field recurrence rate.

The benefit of adjuvant therapy in resected IHCC will depend on appropriate patient selection. Consequently, for producing positive effect about adjuvant RT after R0 resection in IHCC, RT candidates should be carefully selected in patients with recurrence factors, especially patients with a tumor size larger than $5 \mathrm{~cm}$.

\section{Conclusion}

For cholangiocarcinomas, the tumor location and resectability remain prime factors in disease control, whereas adjuvant chemotherapy and radiation remain more controversial. However, surgical resection alone is not sufficient for IHCC despite curative resection. We suggest that patients with tumor size larger than $4 \mathrm{~cm}, \mathrm{LN}$ metastasis, and multiple tumors could be considered about receiving adjuvant RT for control of locoregional recurrence after R0 resection. However, patients with a tumor size larger than $5 \mathrm{~cm}$ should be carefully selected as adjuvant RT candidates. Further studies investigating the subset of high-risk recurrence groups as well as optimal adjuvant therapy for IHCC are needed.

\section{Competing interests}

The authors declare that they have no competing interests.

\section{Authors' contributions}

EG participated in the study design, collected and interpreted data, and prepared the manuscript. JWJ was involved in the study design, coordinated the study, and carried out hepatectomies. JMK assisted with the study design, collected and interpreted data, performed statistical analysis, and carried out hepatectomies. HCP participated in the study design, coordinated the study, and revised the manuscript. JIY revised the manuscript. SHJ collected and interpreted data. JWJ and HCP contributed equally as corresponding authors. All authors read and approved the final manuscript.

\section{Author details}

'Department of Critical Care Medicine, Sungkyunkwan University School of Medicine, 50 Irowndong, Gangnam-gu, Seoul 135-170, South Korea.

${ }^{2}$ Department of Surgery, Samsung Medical Center, Sungkyunkwan University School of Medicine, 50 Irwon-dong, Gangnam-gu, Seoul 135-170, South Korea. ${ }^{3}$ Department of Radiation Oncology, Samsung Medical Center, Sungkyunkwan University School of Medicine, 50 Irwon-dong, Gangnam-gu, Seoul 135-170, South Korea.

Received: 26 April 2015 Accepted: 25 June 2015

Published online: 29 July 2015

\section{References}

1. Anderson CD, Pinson CW, Berlin J, Chari RS. Diagnosis and treatment of cholangiocarcinoma. Oncologist. 2004;9:43-57.

2. Yang J, Yan LN. Current status of intrahepatic cholangiocarcinoma. World J Gastroenterol. 2008;14:6289-97.

3. Malhi H, Gores GJ. Cholangiocarcinoma: modern advances in understanding a deadly old disease. J Hepatol. 2006;45:856-67.

4. Endo I, House MG, Klimstra DS, Gonen M, D'Angelica M, Dematteo RP, et al. Clinical significance of intraoperative bile duct margin assessment for hilar cholangiocarcinoma. Ann Surg Oncol. 2008;15:2104-12.

5. McGlynn KA, Tarone RE, El-Serag HB. A comparison of trends in the incidence of hepatocellular carcinoma and intrahepatic cholangiocarcinoma in the United States. Cancer Epidemiol Biomarkers Prev. 2006;15:1198-203.

6. Shaib Y, El-Serag HB. The epidemiology of cholangiocarcinoma. Semin Liver Dis. 2004;24:115-25.

7. Puhalla H, Schuell B, Pokorny H, Kornek GV, Scheithauer W, Gruenberger T. Treatment and outcome of intrahepatic cholangiocellular carcinoma. Am J Surg. 2005;189:173-7.

8. Endo I, Gonen M, Yopp AC, Dalal KM, Zhou Q, Klimstra D, et al. Intrahepatic cholangiocarcinoma: rising frequency, improved survival, and determinants of outcome after resection. Ann Surg. 2008;248:84-96.

9. Yedibela S, Demir R, Zhang W, Meyer T, Hohenberger W, Schonleben F. Surgical treatment of mass-forming intrahepatic cholangiocarcinoma: an 11-year Western single-center experience in 107 patients. Ann Surg Oncol. 2009;16:404-12.

10. Nakeeb A, Pitt HA, Sohn TA, Coleman J, Abrams RA, Piantadosi S, et al. Cholangiocarcinoma. A spectrum of intrahepatic, perihilar, and distal tumors. Ann Surg. 1996;224:463-73. discussion 473-465. 
11. Lang H, Sotiropoulos GC, Fruhauf NR, Domland M, Paul A, Kind EM, et al. Extended hepatectomy for intrahepatic cholangiocellular carcinoma (ICC): when is it worthwhile? Single center experience with 27 resections in 50 patients over a 5-year period. Ann Surg. 2005;241:134-43.

12. Taylor-Robinson SD, Toledano MB, Arora S, Keegan TJ, Hargreaves S, Beck A, et al. Increase in mortality rates from intrahepatic cholangiocarcinoma in England and Wales 1968-1998. Gut. 2001;48:816-20.

13. Choi SB, Kim KS, Choi JY, Park SW, Choi JS, Lee WJ, et al. The prognosis and survival outcome of intrahepatic cholangiocarcinoma following surgical resection: association of lymph node metastasis and lymph node dissection with survival. Ann Surg Oncol. 2009;16:3048-56.

14. Todoroki T. Chemotherapy for bile duct carcinoma in the light of adjuvant chemotherapy to surgery. Hepatogastroenterology. 2000;47:644-9.

15. Takada $T$, Amano H, Yasuda H, Nimura $Y$, Matsushiro $T$, Kato H, et al. Is postoperative adjuvant chemotherapy useful for gallbladder carcinoma? A phase III multicenter prospective randomized controlled trial in patients with resected pancreaticobiliary carcinoma. Cancer. 2002;95:1685-95.

16. Bektas H, Yeyrek C, Kleine M, Vondran FW, Timrott K, Schweitzer N, et al. Surgical treatment for intrahepatic cholangiocarcinoma in Europe: a single center experience. J Hepatobiliary Pancreat Sci. 2014;22(2):131-7. doi:10.1002/jhbp.

17. Pitt HA, Nakeeb A, Abrams RA, Coleman J, Piantadosi S, Yeo CJ, et al. Perihilar cholangiocarcinoma. Postoperative radiotherapy does not improve survival. Ann Surg. 1995;221:788-97. discussion 797-788.

18. Miyazaki M, Ito H, Nakagawa K, Ambiru S, Shimizu H, Shimizu Y, et al. Aggressive surgical approaches to hilar cholangiocarcinoma: hepatic or local resection? Surgery. 1998;123:131-6.

19. Stein DE, Heron DE, Rosato EL, Anne PR, Topham AK. Positive microscopic margins alter outcome in lymph node-negative cholangiocarcinoma when resection is combined with adjuvant radiotherapy. Am J Clin Oncol. 2005;28:21-3.

20. Cameron JL, Pitt HA, Zinner MJ, Kaufman SL, Coleman J. Management of proximal cholangiocarcinomas by surgical resection and radiotherapy. Am J Surg. 1990;159:91-7. discussion 97-98.

21. Shinohara ET, Mitra N, Guo M, Metz JM. Radiation therapy is associated with improved survival in the adjuvant and definitive treatment of intrahepatic cholangiocarcinoma. Int J Radiat Oncol Biol Phys. 2008;72:1495-501.

22. Alvaro D, Cannizzaro R, Labianca R, Valvo F, Farinati F, Italian Society of G, Italian Association of Hospital G, et al. Cholangiocarcinoma: a position paper by the Italian Society of Gastroenterology (SIGE), the Italian Association of Hospital Gastroenterology (AIGO), the Italian Association of Medical Oncology (AIOM) and the Italian Association of Oncological Radiotherapy (AIRO). Dig Liver Dis. 2010;42:831-8.

23. Murakami Y, Uemura K, Sudo T, Hashimoto Y, Nakashima A, Kondo N, et al, Prognostic factors after surgical resection for intrahepatic, hilar, and distal cholangiocarcinoma. Ann Surg Oncol. 2011;18:651-8.

24. Hanazaki K, Kajikawa S, Shimozawa N, Shimada K, Hiraguri M, Koide N, et al. Prognostic factors of intrahepatic cholangiocarcinoma after hepatic resection: univariate and multivariate analysis. Hepatogastroenterology. 2002:49:311-6.

25. Guglielmi A, Ruzzenente A, Campagnaro T, Pachera S, Valdegamberi A, Nicoli P, et al. Intrahepatic cholangiocarcinoma: prognostic factors after surgical resection. World J Surg. 2009;33:1247-54.

26. Miwa S, Miyagawa S, Kobayashi A, Akahane Y, Nakata T, Mihara M, et al. Predictive factors for intrahepatic cholangiocarcinoma recurrence in the liver following surgery. J Gastroenterol. 2006;41:893-900.

27. Todoroki T, Iwasaki Y, Orii K, Otsuka M, Ohara K, Kawamoto T, et al. Resection combined with intraoperative radiation therapy (IORT) for stage IV (TNM) gallbladder carcinoma. World J Surg. 1991;15:357-66.

28. Mahe M, Romestaing P, Talon B, Ardiet JM, Salerno N, Sentenac I, et al. Radiation therapy in extrahepatic bile duct carcinoma. Radiother Oncol. 1991;21:121-7.

29. Rich TA, Evans DB, Curley SA, Ajani JA. Adjuvant radiotherapy and chemotherapy for biliary and pancreatic cancer. Ann Oncol. 1994;5 Suppl 3:75-80.

30. Bosset JF, Mantion G, Gillet M, Pelissier E, Boulenger M, Maingon P, et al. Primary carcinoma of the gallbladder. Adjuvant postoperative external irradiation. Cancer. 1989;64:1843-7.

31. Jarnagin WR, Ruo L, Little SA, Klimstra D, D'Angelica M, DeMatteo RP, et al. Patterns of initial disease recurrence after resection of gallbladder carcinoma and hilar cholangiocarcinoma: implications for adjuvant therapeutic strategies. Cancer. 2003;98:1689-700.

32. Nathan H, Aloia TA, Vauthey JN, Abdalla EK, Zhu AX, Schulick RD, et al. A proposed staging system for intrahepatic cholangiocarcinoma. Ann Surg Oncol. 2009;16:14-22.

33. Wang Y, Li J, Xia Y, Gong R, Wang K, Yan Z, et al. Prognostic nomogram for intrahepatic cholangiocarcinoma after partial hepatectomy. J Clin Oncol. 2013;31:1188-95

34. Ribero D, Pinna AD, Guglielmi A, Ponti A, Nuzzo G, Giulini SM, et al. Surgical approach for long-term survival of patients with intrahepatic cholangiocarcinoma: a multi-institutional analysis of 434 patients. Arch Surg. 2012;147:1107-13.

35. Farges O, Fuks D, Boleslawski E, Le Treut YP, Castaing D, Laurent A, et al. Influence of surgical margins on outcome in patients with intrahepatic cholangiocarcinoma: a multicenter study by the AFC-IHCC-2009 study group. Ann Surg. 2011;254:824-9. discussion 830

36. Li YY, Li H, LV P, Liu G, Li XR, Tian BN, et al. Prognostic value of cirrhosis for intrahepatic cholangiocarcinoma after surgical treatment. J Gastrointest Surg. 2011;15:608-13.

37. Lang H, Sotiropoulos GC, Sgourakis G, Schmitz KJ, Paul A, Hilgard P, et al. Operations for intrahepatic cholangiocarcinoma: single-institution experience of 158 patients. J Am Coll Surg. 2009;208:218-28.

38. Nakagohri T, Kinoshita T, Konishi M, Takahashi S, Gotohda N. Surgical outcome and prognostic factors in intrahepatic cholangiocarcinoma. World J Surg. 2008;32:2675-80.

\section{Submit your next manuscript to BioMed Central and take full advantage of:}

- Convenient online submission

- Thorough peer review

- No space constraints or color figure charges

- Immediate publication on acceptance

- Inclusion in PubMed, CAS, Scopus and Google Scholar

- Research which is freely available for redistribution 